\title{
Redesign of the Pharmacy Practice Model at a Tertiary Care Teaching Hospital
}

\author{
Heather J Lorimer, Sherry L Lalli, and Sean P Spina
}

\section{INTRODUCTION}

$\mathrm{T}$ he past 3 decades have witnessed the evolution and refinement of clinical pharmacy. A growing body of evidence supports the proposition that ward-based clinical pharmacy practice improves patient care. ${ }^{1-5}$ The work of clinical pharmacists affects the health care system by significantly reducing mortality rates, medication errors, length of stay, and the cost of care. ${ }^{1-5}$ The Blueprint for Pharmacy, a Canada-wide collaborative initiative, has outlined the need for pharmacists to become more patient-centred and outcome-focused. ${ }^{6}$ This approach aligns with the Canadian Society of Hospital Pharmacy (CSHP) 2015 initiative, Targeting Excellence in Pharmacy Practice, which aims to "improve patients' medicationrelated outcomes and safety by advancing pharmacy practice excellence". ' Nationwide, the 2009/2010 Hospital Pharmacy in Canada survey demonstrated that the CSHP 2015 target of increasing the extent to which pharmacists help individual hospital inpatients achieve the best use of medications has not been attained. ${ }^{8}$ To achieve the aforementioned goal, hospitals and other health care institutions need to establish strong clinical pharmacy programs.

Although clinical pharmacy has been in existence for the past 4 decades, there is still a struggle to define the role of the clinical pharmacist. ${ }^{9}$ The daily functions of clinical pharmacists vary greatly among institutions, and discussions continue as to the most effective use of this pharmacy resource. ${ }^{10,11}$ Despite this lack of consensus, it is clear that allowing pharmacists to practise to their full scope will necessitate a shift away from the dispensary-focused model of pharmacy service. ${ }^{11}$ More specifically, a patient-centred, integrated pharmacy practice model would allow nearly all pharmacists to have both distributive and clinical responsibilities. ${ }^{12,13}$ In addition, such a change would allow clinical pharmacists to seamlessly integrate into the interdisciplinary team and thereby improve patient care.
Furthermore, it would offer pharmacists more opportunities for direct patient contact, a requirement for a fulfilling career. ${ }^{14,15}$

This article outlines changes made in a tertiary care teaching hospital to shift the pharmacy practice model from drug distribution with a clinical pharmacy specialist focus to an integrated, patient-centred model.

\section{BACKGROUND TO THE PRACTICE MODEL CHANGE}

The Royal Jubilee Hospital is a 453-bed tertiary care teaching hospital in Victoria, British Columbia, that is part of the Vancouver Island Health Authority (VIHA). Before 2008, the pharmacy practice model at this institution was primarily focused on drug distribution, with limited clinical opportunities. The pharmacists' role was to provide distribution services from the dispensary, where computer access to the centralized drug distribution system was localized. The overall drug distribution system included a pharmacy information system (Cerner, Kansas City, Kansas) linked with various automated drug distribution processes (McKesson, Pittsburgh, Pennsylvania). Clinical services staff available at the time consisted of 4 clinical pharmacy specialists with Doctor of Pharmacy (PharmD) education, each working in one of the following dedicated ward areas: cardiology, intensive care unit, psychiatry, and the Renal Unit. In addition, another pharmacist performed medication reconciliation in the preadmission clinic. The hospital also employed clinical pharmacists, who were able to spend only $3 \mathrm{~h} /$ week on troubleshooting tasks, focusing on pharmacokinetic monitoring and resolving drug therapy problems identified by dispensary staff. This minimal clinical pharmacy coverage meant that physicians often had to dose aminoglycosides and vancomycin independently, rather than pharmacists providing this service. The pharmacy department 
operated with 22.4 full-time equivalent (FTE) pharmacists, with 2 positions vacant at the time (Table 1).

The organization was constructing a new, technologically advanced hospital, which was designed to focus on patientcentred care. Pharmacy leadership recognized that there was an opportunity to improve the pharmacy practice model in the new facility, to align it with the organization's goal of creating a strong interdisciplinary care team.

\section{CHANGES TO PRACTICE MODEL}

\section{Overview}

In 2008, VIHA hired a new pharmacy leadership team at the Royal Jubilee Hospital, consisting of a clinical coordinator (S.P.S.) and an operations coordinator (S.L.L.). Staff pharmacists engaged with the new site leadership team to discuss work flow inefficiencies and lack of job satisfaction. A subsequent decision to introduce pharmacists on additional wards empowered the pharmacists to change the hospital's practice model. The first step in this journey was to create a rotation for a "troubleshooter pharmacist", who would be primarily responsible for resolving drug therapy problems. This pharmacist focused on processing problem orders identified in the dispensary and following patients for whom pharmacokinetic monitoring was required. To foster practice model change, both the staff pharmacists and the pharmacy leadership team embraced and endorsed a culture of clinical pharmacy excellence. At a more mechanical level, the need to create a physically separate clinical office space, to provide segregation from the distribution functions, was also recognized and acted upon.
One of the key steps in implementing the new practice model was to orient pharmacists to clinical practice and to provide a training model for pharmacists joining the organization in the future. Regular education sessions were delivered to the pharmacists to build their confidence in identifying and resolving drug therapy problems in patients with common disease states. In addition, pharmacists were encouraged to attend educational sessions offered through the pharmacy residency program. The advent of hospital-wide wireless computing, coupled with the acquisition of laptops, allowed clinical pharmacists to work from the ward areas, which improved their effectiveness and efficiency. Once these tools were in place and training was complete, in 2009, clinical pharmacists were assigned to selected ward areas (Table 2). A buddy system was used to provide complete coverage on the selected wards. Also at this time, pharmacy planning for the new Patient Care Centre began in earnest, which gave pharmacy leadership an opportunity to introduce the proposed pharmacy practice model to the rest of the interdisciplinary team.

\section{“Lean Design" Project}

In preparation for the new facility, a "lean design" project was completed in $2010 .{ }^{16}$ The project's mandate was to improve efficiency without increasing staff resources. The Pharmacy Department participated in this project with the aim of streamlining overall drug distribution processes. One of the outcomes was the integration, on a trial basis, of a clinical pharmacist and a pharmacy assistant into the interdisciplinary team on the inpatient renal ward. This trial demonstrated the value of having a clinical pharmacist available on the ward to resolve drug therapy problems in a timely manner. During the same timeframe, a new provincial initiative called Care Delivery Table 1. Changes in Pharmacy Service Indicators with Change in Practice
Model at Royal Jubilee Hospital

\begin{tabular}{lccc} 
Fiscal Year* & $\begin{array}{c}\text { Pharmacist } \\
\text { Staffing (FTEs) }\end{array}$ & $\begin{array}{c}\text { Ward Areas } \\
\text { Coveredt }\end{array}$ & $\begin{array}{c}\% \text { of Full Clinical } \\
\text { Coverage for } \\
\text { Hospitalł }\end{array}$ \\
\hline $\begin{array}{c}\text { Immediately before model } \\
\text { change (2008) }\end{array}$ & $\begin{array}{c}22.4 \\
\text { (2 vacancies) }\end{array}$ & $5 / 25$ & 18.4 \\
$2009 / 2010$ & 24.5 & $11.7 \S / 25$ & 37.0 \\
$2010 / 2011$ & 24.0 & $13 / 25$ & 41.1 \\
$2011 / 2012$ & 24.6 & $23 / 239$ & 73.0 \\
& (2 vacancies) & & \\
\hline
\end{tabular}

FTE $=$ full-time equivalent

*Fiscal year runs from April 1 to March 31.

tRefers to ward areas covered on weekdays by clinical pharmacy specialists and clinical pharmacists. In the event of sickness or vacation, pharmacists provide cross-coverage. ¥Percentage of full clinical coverage excludes cross-coverage by pharmacists and time on weekends, nights, or statutory holidays. See text for further explanation. $\S$ Fractional value for number of ward areas covered because expansion of coverage for some areas did not start at the beginning of the fiscal year.

INNew hospital was opened. 


\section{Table 2. Pharmacy Practice Changes Implemented from 2008 to 2012 at Royal Jubilee Hospital}

\begin{tabular}{|c|c|}
\hline \multirow{12}{*}{$\frac{\text { Year }}{2008}$} & Change Implemented \\
\hline & New pharmacy site leadership \\
\hline & $\begin{array}{l}\text { Initiation (by pharmacists) of discussion with pharmacy site leadership regarding job satisfaction and streamlining } \\
\text { of pharmacy operations }\end{array}$ \\
\hline & Initial re-orientation of pharmacists toward clinical practice \\
\hline & Designation of small office space for clinical pharmacists, physically separate from dispensary \\
\hline & Identification of need for a Pharmacist Clinical Worksheet \\
\hline & $\begin{array}{l}\text { Adjustment of pharmacist rotations to include a "trouble-shooter pharmacist" to provide basic clinical } \\
\text { pharmacy services }\end{array}$ \\
\hline & Conversion from desktop PCs to mobile laptops for clinical pharmacists \\
\hline & Initiation of culture of clinical pharmacy excellence \\
\hline & Ward areas covered: \\
\hline & - By clinical pharmacist: preadmission clinic (for medication reconciliation) \\
\hline & - By clinical pharmacy specialists: cardiology, intensive care unit, psychiatry, Renal Unit \\
\hline \multirow[t]{4}{*}{2009} & Decrease of pharmacist resources in the dispensary and increase of resources on wards \\
\hline & Additional ward areas covered: \\
\hline & - By clinical pharmacist: surgery, heart health, palliative care, respiratory therapy, clinical teaching unit, \\
\hline & $\begin{array}{l}\text { and Renal Unit } \\
\text { - By clinical pharmacy specialists: emergency }\end{array}$ \\
\hline \multirow[t]{9}{*}{2010} & Completion of pharmacy's "lean design" review \\
\hline & Participation by pharmacy leadership in provincial Care Delivery Model Redesign \\
\hline & Design of tracking database for rDTPs \\
\hline & Assignment of larger office space for clinical pharmacists (for effective collaboration) \\
\hline & Design of clinical e-mail inbox system \\
\hline & Piloting of new clinical pharmacy practice model, with clinical pharmacist and pharmacy assistant on ward \\
\hline & Creation of clinical pharmacy support assistant position for Renal Unit (for medication reconciliation) \\
\hline & Additional ward area covered: \\
\hline & - By clinical pharmacist: atrial fibrillation clinic \\
\hline \multirow[t]{9}{*}{2011} & Extension of dispensary hours of operation \\
\hline & Opening of new hospital (Patient Care Centre) \\
\hline & Implementation of Vocera communication system \\
\hline & Implementation of dedicated ward space, with dual-monitor workstations, for clinical pharmacists \\
\hline & Transition to ward-based order verification \\
\hline & Roll-out of rDTP database to all clinical pharmacists \\
\hline & Reporting of data from rDTP database to senior administration \\
\hline & $\begin{array}{l}\text { Extension of clinical pharmacist coverage to all hospital ward areas, with continuation of coverage by clinical } \\
\text { pharmacy specialists where already in place }\end{array}$ \\
\hline & $\begin{array}{l}\text { Adjustment of rotations for non-residency-trained pharmacists to increase their clinical time (medication } \\
\text { reconciliation in preadmission clinic, rehabilitation ward) }\end{array}$ \\
\hline \multirow[t]{5}{*}{2012} & Implementation of PharmNet Order Imaging system \\
\hline & Piloting of clinical pharmacy assistants in Patient Care Centre \\
\hline & Switch to smartphone (iPhone4) as standard communication device \\
\hline & Initiation of Clinical Chit Chat meetings \\
\hline & Piloting of Pharmacist Clinical Worksheet \\
\hline
\end{tabular}

Model Redesign showed that activities being performed by all front-line care providers had drifted away from their defined scopes of practice. The provincial initiative focused on interdisciplinary care, which allowed pharmacists to participate as welcomed members of the team. A desired outcome of the process was to validate the reassignment of pharmacists to the wards, away from the dispensary. Survey feedback obtained from members of the interdisciplinary team during the renal ward trial demonstrated that the clinical pharmacist's impact was valuable, and the trial resulted in requests for the expansion of clinical pharmacy services to other areas.

\section{Technology and Communication}

Traditionally, it has been a challenge to concretely document the patient care benefits of clinical pharmacists' involvement. ${ }^{17}$ Resolution of this issue was recognized as a priority during development of the VIHA Clinical Pharmacy Strategic Plan. Resolved drug therapy problems (rDTPs) was chosen as the surrogate indicator of pharmacists' contributions to patient care. A database for tracking rDTPs, based on Access software (Microsoft, Seattle, Washington), was designed and developed at the Royal Jubilee Hospital, in collaboration with 
the University of Victoria, to allow collection and reporting of drug therapy problems resolved by pharmacists. In addition, the database gave pharmacists the ability to generate a best possible medication history for each patient, to facilitate medication reconciliation on admission and discharge.

As pharmacists' work shifted away from the dispensary, the need for effective communication became evident. The initial step in improving communication was to create a common e-mail inbox, accessible to all pharmacy staff, where pharmacists could convey ongoing clinical issues to their colleagues to improve continuity of care. With the opening of the new hospital in March 2011, there were further improvements in communication technology, including introduction of a proprietary real-time communication system (Vocera, San Jose, California) and extensive video-conferencing capability throughout the hospital. The Vocera system is now the primary communication method within the interdisciplinary team. Other technological changes included the transition, in 2012, from pagers to smartphones (iPhone 4, Apple Inc) as the standard communication device for all VIHA pharmacists. Additional improvements in technology included installation of pharmacist-dedicated, dual-monitor workstations on the wards, which allow for better ward-based order verification. In January 2012, PharmNet Order Imaging (POI; Cerner, Kansas City, Kansas), an integrated imaging system for patient care orders, was implemented to allow remote order verification by pharmacists. This system made pharmacists' traditional use of paper orders obsolete. Instead, pharmacists now use the dual-screen monitors, viewing any order of interest on one screen and verifying the order on the other. All of these technological improvements permitted expansion of pharmacist coverage to all 23 ward areas ( 453 beds) at the Royal Jubilee Hospital in 2012.

\section{Education}

The outcome of all these process changes is that clinical pharmacists, who in 2008 were working almost exclusively in the dispensary, are now able to spend $80 \%$ of their shifts working on the wards. In addition, the job functions of nonresidency-trained pharmacists have changed significantly. They now work within the preadmission clinic, where they perform medication reconciliation, and practise clinical pharmacy on the rehabilitation ward. In an effort to provide ongoing learning opportunities in clinical pharmacy practice, these nonresidency-trained pharmacists continue to work under the mentorship of a clinical pharmacy specialist on the psychiatry wards. To build upon the framework of a collaborative teaching environment, "Clinical Chit Chat" meetings were instituted, where pharmacists can share their clinical experiences in a structured format, thus strengthening the team dynamic.

\section{Additional Initiatives}

To get closer to the overall vision of providing clinical pharmacy services to all patients, various other initiatives have been trialled to increase the amount of time that pharmacists are available for clinical activities. One trial with positive results involved the reassignment of tasks associated with obtaining and verifying patients' own medications, as well as assigning the dispensing of "pass medications" to clinical pharmacy assistants, rather than clinical pharmacists. These changes led to a significant increase in the time that clinical pharmacists could spend with patients (equivalent to $50 \mathrm{~h} /$ week). To support clinical pharmacy excellence, the need for a Pharmacist Clinical Worksheet was recognized; the resulting worksheet was designed to easily identify patients at the greatest risk of clinically significant drug-related harm. Anecdotally, use of this worksheet increased the time available for clinical activities by decreasing the time required to identify and prioritize patients who would most benefit from clinical pharmacy services. The Pharmacist Clinical Worksheet lists all patients under each pharmacist's care, including information about their demographic characteristics, serum creatinine level, serum drug levels, antibiotic therapy (with start and stop dates), regularly scheduled IV medications, and drugs typically requiring pharmacokinetic monitoring. Use of the worksheet will be fully implemented in 2013.

\section{IMPLICATIONS}

The role of clinical pharmacy at Royal Jubilee Hospital has grown impressively over the past 4 years. The number of ward areas with clinical coverage has increased progressively, from 5 (20\%) of 25 ward areas in 2008 to all 23 ward areas (100\%) in 2011 (Table 1). The percentage of full clinical coverage throughout the hospital was calculated retroactively from scheduling data. Full clinical coverage was defined as assignment of a pharmacist to a single ward on a particular day. Assignment of a pharmacist to cover more than one ward was considered to represent full coverage for one ward and troubleshooting coverage for the other wards. The percent coverage indicates the proportion of clinical shifts that were filled. The goal of $100 \%$ clinical coverage has not yet been attained because of limitations on available staff to provide backfill for illness and vacation relief. Nonetheless, the percentage of hospital ward areas with clinical coverage increased from $18.4 \%$ in 2008 to $73.0 \%$ in $2011 / 2012$ (Table 1). Although clinical cross-coverage for each ward was available when pharmacists were on vacation, this approach did not provide the same level of care as a dedicated clinical pharmacist, and such coverage was therefore not included in calculating the percent clinical coverage. 
In the new practice model, clinical pharmacists participate in daily interdisciplinary care rounds on the unit; use a clinical worksheet, in addition to the standard order verification process, to identify patients requiring intervention; work collaboratively with the team to resolve drug therapy problems; participate in admission and discharge medication reconciliation; document interventions in patients' health care records; and counsel patients about their medications before discharge. The clinical pharmacist prioritizes these activities according to the daily workload.

The number of pharmacist FTEs remained relatively stable during implementation of the new practice model (Table 1). Changes in the practice model were made possible by improvements in the efficiency of the drug distribution system, which meant that pharmacist resources could be reassigned from the dispensary to the wards. More specifically, by forwarding problem orders requiring follow-up and clarification to the clinical pharmacists at the ward level, the dispensary has continued to operate efficiently but with fewer pharmacists. Before 2008, pharmacists were spending almost all of their time dealing with medication distribution functions. Under the new model, $79 \%$ of clinical time is now spent on purely clinical activities and $21 \%$ on distribution activities at the ward level (based on pharmacists' self-reported data for 15-min intervals over 44 clinical shifts). Although the role of clinical pharmacists would ideally have been completely separated from distribution tasks, the compromise approach of bringing distribution tasks to the ward level has allowed realization of the vision for a new pharmacy practice model.

\section{BARRIERS OVERCOME}

The journey toward creating an environment of clinical excellence has had certain challenges. Once pharmacists' initial skepticism of the plan was overcome, it was necessary to improve their comfort and confidence working in the new clinical practice model. An additional challenge was the requirement to gain acceptance from the health care team and the organization as a whole. Finding appropriate workspace, addressing competing organizational priorities, and accommodating delays in technological upgrades were also required.

\section{Pharmacists' Comfort with Clinical Practice}

During initial discussions about the improvements needed in the system, pharmacists identified the need to streamline distribution inefficiencies. When pharmacy leadership presented the vision of a new clinical practice model, the pharmacists were initially skeptical that it would succeed. However, the leadership team empowered pharmacy staff members to participate in redesigning the practice model and thereby to take ownership of the change. During the transition to the new clinical practice model, it became apparent that most of the pharmacists lacked the confidence to practise in a clinical model, because of years spent performing principally dispensary duties. To address this barrier to success, pharmacists were initially assigned a lower case load than would be assigned after full implementation, to allow them time to reacquaint themselves with clinical practice. Further support was provided through regular mentorship by senior clinicians. The clinical pharmacists worked in organizational groupings called "pods" to ensure coverage of multiple ward areas and a system of overlap for discussion of clinical concerns. The pod structure facilitated learning and improved pharmacists' comfort with their clinical practice area.

\section{Interdisciplinary Team Culture}

As clinical pharmacists were introduced to the interdisciplinary team, it was evident that their role was poorly understood by other members of the team. However, the daily, visible presence of ward-based clinical pharmacists fostered a change in culture toward embracing the profession of pharmacy on the ward. Whereas previous interactions had been by telephone, the ability to speak face to face and discuss issues on the ward improved communication within the team. At the same time, VIHA as a whole was participating in the provincial Care Delivery Model Redesign, which encouraged full integration of pharmacists within the interdisciplinary team. By establishing a clinical pharmacy model on the wards, an environment was created in which clinical pharmacists have become an essential part of the team, and their involvement in patient care is regularly requested by other health care professionals.

\section{Insufficient Workspace and Conflicting Organizational Priorities}

To ensure that clinical pharmacists became an established presence on the ward, it was identified that a designated workspace would be required. The pharmacy leadership team worked with the organization to garner support in creating such a dedicated workspace in every ward of the new Patient Care Centre, based on the need to deploy staff to the area regularly.

As noted above, the lean design project, completed in 2010, had a mandate to stay within the existing level of staffing when changes in work processes were proposed. The efficiencies gained were to be funnelled into the new clinical vision of ward-based pharmacy services. Two months before implementation of the clinical vision, the organization mandated that the pharmacy department's hours of operation be extended. However, without additional staff, such an expansion of services threatened the department's ability to implement the 
new practice model. The pharmacy site leadership team revised the implementation plan (adjusting work schedules and reviewing processes to ensure that each task was assigned to the most appropriate professional), which allowed hours of operation to increase without additional staff.

\section{Delayed Implementation of Technology}

Ideally, to ensure the success of the vision for clinical pharmacy practice, technological advancements should have been implemented before the change in practice. For VIHA as a whole, it was anticipated that the POI system would be operational by the time the clinical pharmacists were deployed to the wards. However, when the POI system was delayed by more than a year, the decision was made to continue with the planned deployment of clinical pharmacists to the wards and to develop a workaround to ensure they could function in both clinical and distribution modes. This interim workaround, though inefficient, allowed pharmacists to continue integrating the Care Delivery Model Redesign process without loss of momentum.

\section{FUTURE PLANS}

Given the overwhelming success of this model change at Royal Jubilee Hospital, it is currently being expanded to other hospitals within the VIHA. The change in practice model has ensured access to a clinical pharmacist for all patients at this large tertiary care teaching hospital. To implement this vision of clinical pharmacy services at other VIHA hospitals, certain adjustments may be required. At the Royal Jubilee Hospital, development and implementation of formal roles for clinical pharmacy assistants and technicians will be required to further support the clinical pharmacists on the wards in practising to their full scope. Because it is no longer sufficient for pharmacists to be involved in care on weekdays only, ${ }^{12}$ further expansion efforts should focus on the provision of high-quality clinical pharmacy services 7 days per week.

\section{CONCLUSIONS}

At the Royal Jubilee Hospital in Victoria, British Columbia, we implemented a variety of strategies to allow pharmacists to practise on patient care units. Necessary elements of the change in practice model included engaging staff pharmacists in the vision, evaluating current processes against "lean design" principles, improving communication, committing to pharmacist education, implementing digital imaging software, and obtaining support from leadership, both within the pharmacy and at the level of health authority.

Any practice change represents an evolution in care processes and usually requires compromise. To make a practice model change of this magnitude, leadership must stay focused on the vision and must make necessary revisions to overcome obstacles as they arise, rather than abandoning the entire initiative. It is essential to be aware of organizational priorities and align practice change with those priorities, for a higher probability of success. Leadership must also be sensitive to practice changes occurring in other health care professions and the possibility of integrating within their process change. The timing of a change in practice model will never be perfect. As Mahatma Gandhi said, "The future depends on what you do today". ${ }^{18}$

\section{References}

1. Gillespie U, Alassaad A, Henrohn D, Garmo H, Hammarlund-Udenaes $\mathrm{M}$, Toss $\mathrm{H}$, et al. A comprehensive pharmacist intervention to reduce morbidity in patients 80 years or older: a randomized controlled trial. Arch Intern Med. 2009;169(9):894-900.

2. Bond CA, Raehl CL, Franke T. Interrelationships among mortality rates, drug costs, total cost of care, and length of stay in United States hospitals: summary and recommendations for clinical pharmacy services and staffing. Pharmacotherapy. 2001;21(2):129-41.

3. Bond CA, Raehl CL. Clinical pharmacy services, pharmacy staffing, and hospital mortality rates. Pharmacotherapy. 2007;27(4):481-93.

4. Bond CA, Raehl CL, Franke T. Clinical pharmacy services and hospital mortality rates. Pharmacotherapy. 1999;19(5):556-64.

5. Bond CA, Raehl CL, Franke T. Clinical pharmacy services, hospital pharmacy staffing, and medication errors in United States hospitals. Pharmacotherapy. 2002;22(2):134-47.

6. Blueprint for pharmacy: the vision for pharmacy. Ottawa (ON): Canadian Pharmacists Association; 2011 [cited 2012 Jun 1]. Available from: http://blueprintforpharmacy.ca/about

7. CSHP 2015 frequently asked questions. Ottawa (ON): Canadian Society of Hospital Pharmacists; 2012 [cited 2012 Jun 1]. Available from: www.cshp.ca/programs/cshp2015/faq/index_e.asp

8. Babich M, Bornstein C, Bussières JF, Hall K, Harding J, Lefebvre P, et al., editors. Hospital pharmacy in Canada 2009/2010 report. Eli Lilly; 2010 [cited 2012 Jun 1]. Available from: www.lillyhospitalsurvey.ca/hpc2/ content/2010_report/2009_2010_full_E.pdf

9. Gonzalez LS 3rd. What are pharmacists, and what do they do? Am J Health Syst Pharm. 2005;62(19):2039-40.

10. Bush PW, Ashby DM, Guharoy R, Knoer S, Rough S, Stevenson JG, et al. Pharmacy practice model for academic medical centers. Am J Health Syst Pharm. 2010;67(21):1856-61.

11. Zellmer WA. Seeking "better ways" in hospital pharmacy. Am J Health Syst Pharm. 2009;66(8):713.

12. Woods TM, Lucas AJ, Robke JT. Making a case for a patient-centered integrated pharmacy practice model. Am J Health Syst Pharm. 2011; 68(3):259-63.

13. Pedersen CA, Schneider PJ, Scheckelhoff DJ. ASHP national survey of pharmacy practice in hospital settings: dispensing and administration2008. Am J Health Syst Pharm. 2009;66(10):926-46.

14. Hertig JB, Kelley KA, Jordan TA, Tubbs CR, Casper KA, Nahata MC. Advancing the pharmacy practice model: survey of new practitioner attitudes and opinions. Hosp Pharm. 2011;46(3):180-95.

15. Hertig J. New practitioners and the pharmacy practice model initiative: our opportunity to define the future. Am J Health Syst Pharm. 2011;68(12):1074-6.

16. Krafcik JF. Triumph of the lean production system. MIT Sloan Manag Rev. 1988;30(1):41.

17. Fowler A, Campbell D. Benchmarking and performance management in clinical pharmacy. Int J Oper Prod Manag. 2001;21(3):327-50.

18. Quotations from Mahatma Gandhi. In: Goodreads [website]. Goodreads Inc; 2012 [cited 2012 Jun 1]. Available from: www.goodreads.com/ quotes $/$ search?page=1\&q=Gandhi\&utf8=\%E2\%9C\%93

Heather J Lorimer, BSC(Microbiology), was a pharmacy summer student with the Royal Jubilee Hospital, Victoria, British Columbia, and is currently a pharmacy student in the bachelor's program (candidate for graduation in 2013), Faculty of Pharmaceutical Sciences, The University of British Columbia, Vancouver, British Columbia. 
Sherry L Lalli, BSC(Pharm), ACPR, is the Pharmacy Site Coordinator at Royal Jubilee Hospital, Vancouver Island Health Authority, Victoria, British Columbia.

Sean P Spina, BSc(Pharm), ACPR, PharmD, is the Pharmacy Clinical Coordinator for Royal Jubilee Hospital, Vancouver Island Health Authority, Victoria, British Columbia. He is also a Clinical Assistant Professor, Faculty of Pharmaceutical Sciences, The University of British Columbia, Vancouver, British Columbia.

Competing interests: For work on topics unrelated to this article, Sean Spina has received unrestricted grant support from Jamieson Laboratories, payment for lectures from Pfizer, and payment for development of continuing education from the University of British Columbia (Continuing Pharmacy Education) and the Canadian Society of Hospital Pharmacists.
Address correspondence to:

Dr Sean Spina

Department of Pharmacy

Royal Jubilee Hospital (RB 219)

1952 Bay Street

Victoria BC V8R 1J8

e-mail: Sean.Spina@viha.ca

Acknowledgements

We would like to thank all members of Royal Jubilee Hospital Department of Pharmacy and collaborative team members for their effort and patience during transformation of our practice model. This change would not have been possible without the support of the VIHA Pharmacy Executive: Michele Babich, Dr Richard Bachand, and Winnie Lam. Société canadienne des pharmaciens d'hôpitaux

\section{Membership Year 2012/2013 (July 1, 2012 - June 30, 2013)}

Are you a current member of the Canadian Society of Hospital Pharmacists?

CSHP has celebrated 65 years of inspiration and leadership among a rapidly growing network of over 3000 pharmacy colleagues. As a member of CSHP, you will have access to resources and opportunities that will enhance your career and help impact the direction of healthcare in Canada. Your participation in CSHP 2015, an initiative challenging all pharmacists to improve patient medication outcomes and safety through advancing pharmacy practice excellence by the year 2015 , is a prime example.

For more information about CSHP, please visit us at www.cshp.ca. We invite you to reap the benefits of an affordable membership with CSHP.

Membership Enquiries: Please contact Membership Services

Bus.: (613) 736-9733 Ext. 222 Fax: (613) 736-5660Ｅmail: membershipservices@cshp.ca 